\title{
CARDIAC AUTONOMIC CONTROL IN ELITE JUVENILE CYCLISTS
}

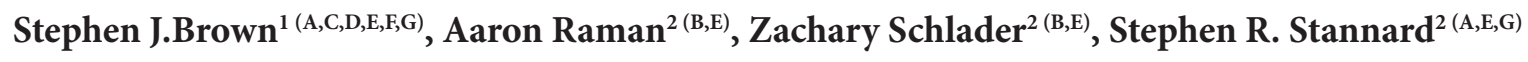 \\ ${ }^{1}$ School of Sport and Exercise, Institute of Food, Nutrition and Human Health, Massey University Albany, \\ Auckland, New Zealand \\ ${ }^{2}$ School of Sport and Exercise, Institute of Food, Nutrition and Human Health, Massey University, Palmerston \\ North, New Zealand
}

\begin{abstract}
Introduction: Frequency domain analysis of heart rate variability (HRV) may potentially identify the dominance exhibited by different branches of the autonomic nervous system. Autonomic contribution to cardio-deceleration following exercise has been studied in adults; however, no data are available for endurance trained juvenile athletes.

Aim: The aim of this study was to evaluate the association between the increase in heart rate following exercise and any change in HRV frequency components in trained juveniles.

Methods: A 6 min resting ECG (lead 2) was recorded from trained juvenile cyclists (23 male, 7 female, mean age 14.7 years), before (Pre) and after (Post) incremental exercise to volitional exhaustion on a stationary cycle ergometer. Each subject performed a progressive ramp protocol where power increased by $20 \mathrm{~W} \mathrm{~min}{ }^{-1}$, with initial power set at $60 \mathrm{~W}$ for females and $100 \mathrm{~W}$ for males. Oxygen uptake $\left(\dot{\mathrm{VO}}_{2}\right)$ was measured using breath-by-breath techniques. ECG was analysed in both time and frequency domains using commercially available software.

Results: Mean $\dot{\mathrm{VO}}_{2}$ max was $75.5 \mathrm{ml} \mathrm{Kg}^{-1} \mathrm{~min}^{-1}$, and mean power at $\dot{\mathrm{VO}}_{2}$ max was $440 \mathrm{~W}$. The R-R interval SD was lower following exercise (Pre: $86 \mathrm{~ms}$ vs. Post: $36 \mathrm{~ms}, P<0.01$ ). Normalised high frequency HRV was lower (Pre: 36.5 vs. Post: 18.7 , $P<0.01$ ) and normalised low frequency HRV was higher (Pre: 58.5 vs. Post: $75.0, P<0.01$ ) following exercise.

Conclusions: In elite juvenile athletes there were no associations between exercise-induced changes in high frequency variability and heart rate $\left(R^{2}=0.073\right)$. This suggests that in elite juveniles, the heart may be less sensitive to vagal influences - a suggestion further supported by the relatively high pre-exercise resting heart rates with normal high frequency variability.
\end{abstract}

Key words: autonomic nervous system, heart rate variability, $\dot{\mathrm{V}} \mathrm{O}_{2}$ max, recovery

\section{Introduction}

Inherent rhythmicity in neural activity directed at the sinus node contributes to heart rate variability (HRV) and may indicate cardiac sympatho-vagal balance [1-3]. Reduced variability may suggest increased mortality [4], although regular exercise has been shown to affect HRV [5].

Post exercise cardiac autonomic control has been studied in untrained $[6-8]$ and trained $[9,10]$ subjects, although predominantly in adults. For example, a decrease in HRV in both time and frequency domains was reported following 1 hour of high intensity interval training [9]. Others [10] reported a post-exercise shift in autonomic balance from vagal to sympathetic dominance following exercise at either $50 \%$ or $80 \%$ maximal oxygen uptake. An increased high frequency component of HRV during recovery from 40 min cycling exercise at $80 \%$ maximal oxygen uptake was reported in males, and regular endurance training reduced postexercise HRV in the time domain when compared to untrained males [11]. Others [12] reported a decrease in total spectral power and a reduced high frequency component of HRV following a single bout of high intensity exercise in well trained older athletes, a finding similarly reported in young adults [13].

Both physical activity and age have been shown to influence cardiac autonomic control. For example, in older subjects, heart rate variability (HRV) in both time and frequency domains increased with 3 months of physical training, and a weak correlation was reported between changes in exercise capacity and changes in HRV spectral power [14]. However, others [15] reported no change in resting heart rate, total HRV spectral power, or low and high frequency components of HRV, following 8 weeks aerobic training in elderly subjects. Similarly, no changes in the high and low frequency components of HRV were reported following 1 year of aerobic training in elderly males [16], although training increased HRV total power and power in the very low frequency range. When a longer exercise intervention was carried out, Uutisalo et al [17] reported that the only significant change in 
HRV was a decrease in the LF component. In both young and older adults, a training-induced increase in resting HRV was reported [18], although no measures in the frequency domain were reported.

Higher resting heart rates in children may limit vagal withdrawal following exercise, however, there are no data regarding HRV during recovery from exercise in well trained juveniles. The originality of the current study is that we have focussed on elite juvenile cyclists - currently there are no data on post exercise changes in heart rate variability for this group. Therefore, the current study examined post exercise HRV in a cohort of well trained juvenile athletes. It was hypothesized that:

1. A stable tachycardia would be evident during recovery from high intensity exercise;

2. This would be accompanied by reduced HRV measured in both time and frequency domains.

3. There would be a close association between the increase in heart rate following exercise and the change in HRV frequency components.

\section{Materials and methods}

All procedures were approved by the Massey University Human Ethics Committee (MUHEC) Southern, and parental/guardian consent was obtained for all subjects. All subjects were under 16 years of age; therefore all required the written permission of a parent or guardian to participate in this study. Additionally during testing, we required the assignee to be present throughout, along with a suitably qualified first-aider with a current cardio-pulmonary resuscitation certificate. Consent was conditional on the satisfactory understanding of information presented both orally and in written format which detailed the procedures of the testing - this information was presented to the subject and his/her assignee. For inclusion in the study, potential subjects were required to meet the following criteria:

1. Had participated in cycle races or triathlon at a minimum of local club standard for at least 1 year;

2. Had represented school, club, area, or Nation, in a cycle or cycling related event (e.g. triathlon) in the preceding 12 months;

3. Had participated in endurance training for a minimum of 12 months, with a frequency of at least three sessions per week, with the average session duration of $>$ 1hour.

Training status of the subjects was not assessed (e.g. with the collection of data from a training diary). We were reliant on the accuracy of the information given by the subject and/or parent/guardian prior to consent.

Trained juveniles ( $n=30,23$ males, 7 females), mean (SD) age 14.8 (1.3) years, mass 61.95 (10.68) $\mathrm{Kg}$ and height $170.5(8.3) \mathrm{cm}$, participated in this study. For the exercise test, all subjects arrived at the laboratory following a $4-6 \mathrm{~h}$ fast, and all testing took place in a built-for-purpose Human Performance Lab with appropriate temperature and ventilation control. Before and after exercise, blood pressure was measured in duplicate manually using a stethoscope and a sphygmomanometer over the right brachial artery, and all values obtained were within a normal range.

\section{Exercise Protocol}

Subjects performed a single test following a normal period of at least 10 weeks during which subjects maintained their normal training/competition schedules. All exercise was undertaken on an electronically braked Lode Excalibur Cycle Ergometer (Groningen, The Netherlands) set in pedal rate-independent mode. The ergometer was set up to replicate each subject's normal cycling position. Throughout each test, expired gases were analyzed using commercially available cardiopulmonary exercise testing apparatus (Turbofit, Vacumed, California, USA). Using standard algorithms, minute

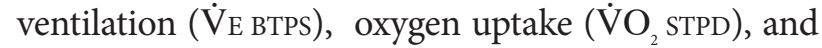
carbon dioxide output ( $\dot{\mathrm{COO}}_{2}$ STPD) were calculated. Heart rate was continuously monitored throughout the test using telemetry (Polar Vantage XL, New Zealand).

At the start of the test, each subject performed a 10 minute warm-up on the ergometer: this was at 60 $\mathrm{W}$ for females, and at $100 \mathrm{~W}$ for males. All subjects performed a continuous ramp protocol at $20 \mathrm{~W}$ per minute until volitional fatigue, with a starting power output equivalent to that of the warm-up. The test was terminated at a point where the subject could no longer perform exercise at the required power output, or at the request of the subject. Throughout the test, verbal encouragement was given. Following the test, each subject performed a 5 minute 'recovery' ride on the ergometer at $50 \mathrm{~W}$.

\section{ECG and HRV recordings}

Before and after exercise, subjects were seated and relaxed for $20 \mathrm{~min}$. Following these periods, a $10 \mathrm{~min}$ continuous electrocardiogram (ECG, limb lead 2, PowerLab + Chart5, AD Instruments, Australia) was recorded while the subject remained seated and relaxed. From the ECG, heart rate and HRV were determined using commercially available software (HRV Module for Chart 5, AD Instruments, NZ). Intervals between adjacent $\mathrm{R}$ waves were detected using a threshold detection of between 0.5 and $1.0 \mathrm{mV}$, and classified as $\operatorname{artifact}(<200 \mathrm{~ms}$ and $>1500 \mathrm{~ms}$ ), ectopic (200 ms to $500 \mathrm{~ms}$, and $1200 \mathrm{~ms}$ to $1500 \mathrm{~ms}$ ), and normal (500 $\mathrm{ms}$ to $1200 \mathrm{~ms}$ ). HRV data were analysed in the time domain using the mean R-R interval, and the standard deviation of the normal mean R-R interval (SD RR). HRV data were analysed in the frequency domain (1,024 FFT) using a Welch averaged period method (histogram bin size $10 \mathrm{~ms}$ ), and banded as Very Low 
Frequency (VLF: $0-0.04 \mathrm{~Hz}$ ), Low Frequency (LF: $0.04-0.15 \mathrm{~Hz}$ ), and High Frequency (HF: $0.15-0.4$ $\mathrm{Hz}$ ). Total power for each spectrum was defined as the area under the spectrum from 0 to $0.5 \mathrm{~Hz}$, and normalised units (nu) for the LF and HF components (which take into account any changes in total spectrum power) were used to calculate the LF: HF ratio.

\section{Statistical analysis}

Pre- vs. Post-exercise data were compared with paired samples Student's $t$-test for samples of equal variance, with significance set at $P<0.05$. The association between exercise-induced changes in HRV frequency components (both LF and HF) and the increase in heart rate following exercise was quantified using linear regression.

\section{Results}

All 30 subjects completed the exercise test satisfactorily, with tests terminated upon volitional fatigue. Mean (SD) power at $\dot{\mathrm{V}} \mathrm{O}_{2} \max$ was 440 (25) W, and mean $\dot{\mathrm{V}} \mathrm{O}_{2}$ max was $75.5(11.3) \mathrm{ml} \mathrm{Kg}^{-1} \cdot \mathrm{min}^{-1}$. On test termination, all subjects had reached a plateau in their oxygen consump-
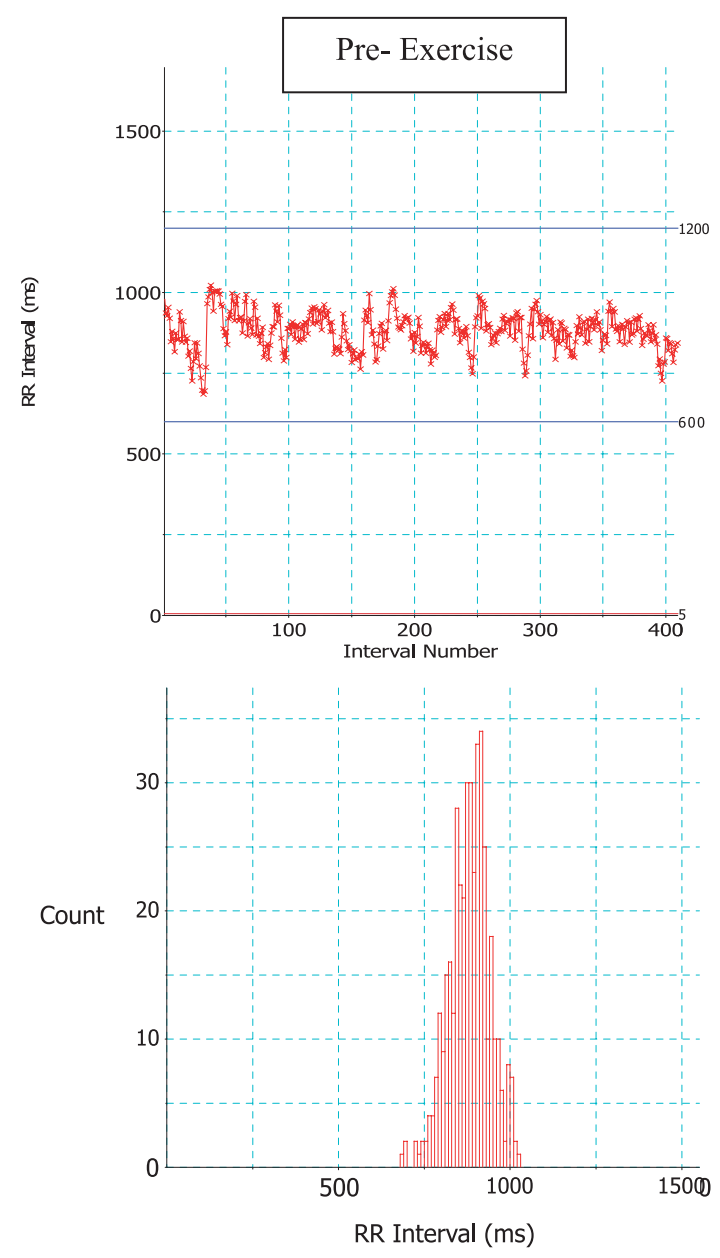

tion, had a respiratory exchange ratio $>1.1$, and could no longer continue to exercise at the same power output.

Ventilation rates were similar during the Pre- and Post-exercise, and were $11.2+/-4.7$ breaths per minute, and $14.3+/-6.2$ breaths per minute respectively $(P>0.05)$. Also, mean tidal volumes were similar during the Pre- and Post-exercise periods, and were $0.7+/-0.2$ $\mathrm{L}$, and $0.9+/-0.3 \mathrm{~L}$ respectively $(P>0.05)$. Given the small (not statistically significant) increases in both ventilation rate and depth during the Post-exercise period, a small increase in minute ventilation was recorded Postexercise (Pre: $7.85 \mathrm{~L} \cdot \mathrm{min}^{-1}$; Post: $12.9 \mathrm{~L} \cdot \mathrm{min}^{-1}, P<0.05$ ).

A representative example of heart rate variability measured in the time domain is shown in figure 1 . In this figure, the upper panels plot successive R-R intervals (y axis) throughout the recording, with the first beat denoted as 'interval 1'. The lower panels are histograms of the R-R interval ( $10 \mathrm{~ms}$ bin size, $\mathrm{x}$ axis). This figure illustrates the greater successive beat-tobeat variation in R-R interval, the more widespread variation in $\mathrm{R}-\mathrm{R}$ intervals, and thus the higher variability before exercise, compared to after exercise. In figure 1, the mean heart rate before exercise was 68
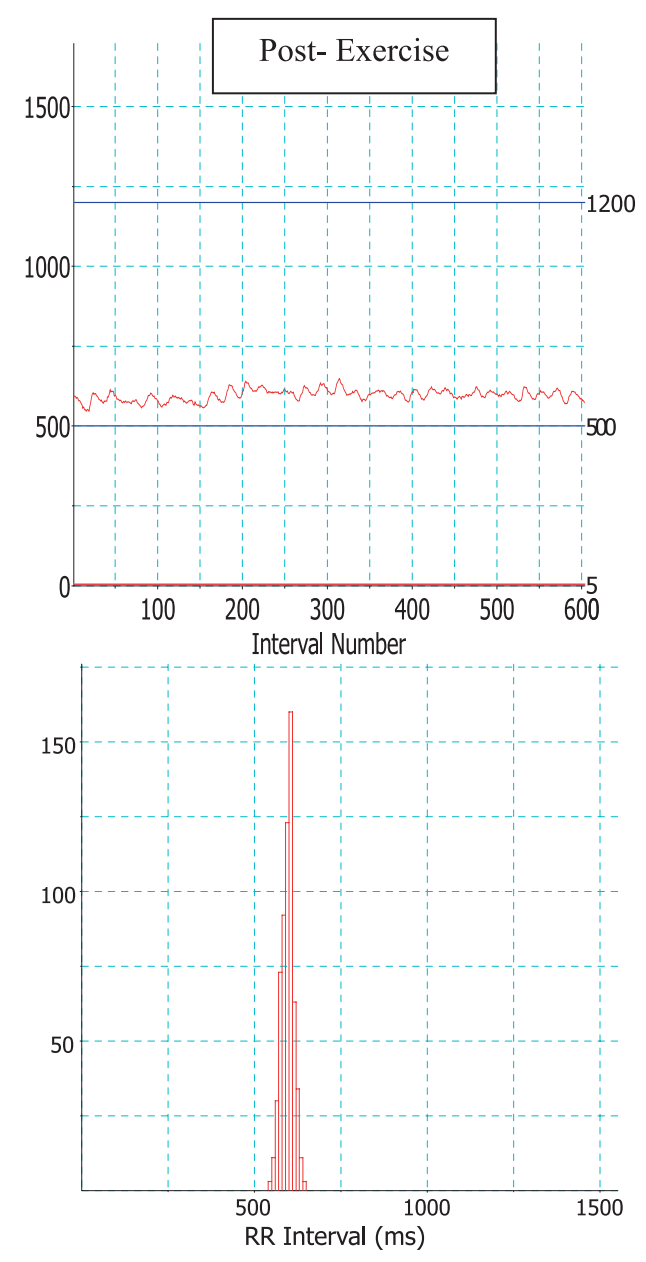
Table 1. Mean (SD) time and frequency domain measures of HRV before and after exercise ( $n=30$, mean age 14.8 years; mean $\left.\dot{V} O_{2} \max 75.5 \mathrm{ml} \mathrm{Kg}^{-1} \cdot \mathrm{min}^{-1}\right)$. All comparisons between Pre and Post, using Student paired sample t-tests, were significantly different $(P<0.01)$

\begin{tabular}{|c|c|c|c|c|c|c|c|}
\hline Pre-exercise & & & & & & & \\
\hline $\begin{array}{c}\text { Mean } \mathrm{HR} \\
\text { beats. } \min ^{-1}\end{array}$ & $\begin{array}{l}\text { SD RR } \\
\text { ms }\end{array}$ & $\begin{array}{c}\text { Total Power } \\
\mathrm{ms}^{2}\end{array}$ & $\begin{array}{c}\mathrm{LF}(0.04-0.15 \mathrm{~Hz}) \\
\mathrm{ms}^{2}\end{array}$ & LF nu & $\begin{array}{c}\mathrm{HF}(0.15-0.4 \mathrm{~Hz}) \\
\mathrm{ms}^{2}\end{array}$ & HF nu & $\mathrm{LF} / \mathrm{HF}$ \\
\hline $\begin{array}{c}71 \\
(10)\end{array}$ & $\begin{array}{c}86 \\
(30)\end{array}$ & $\begin{array}{c}7920 \\
(5202)\end{array}$ & $\begin{array}{c}2825 \\
(1987)\end{array}$ & $\begin{array}{c}58.5 \\
(14.7)\end{array}$ & $\begin{array}{c}1927 \\
(1436)\end{array}$ & $\begin{array}{c}36.5 \\
(13.8)\end{array}$ & $\begin{array}{c}2.04 \\
(1.31)\end{array}$ \\
\hline \multicolumn{8}{|l|}{ Post-exercise } \\
\hline $\begin{array}{c}\text { Mean HR } \\
\text { beats.min }{ }^{-1}\end{array}$ & $\begin{array}{c}\text { SD RR } \\
\text { ms }\end{array}$ & $\begin{array}{c}\text { Total Power } \\
\mathrm{ms}^{2}\end{array}$ & $\begin{array}{c}\mathrm{LF}(0.04-0.15 \mathrm{~Hz}) \\
\mathrm{ms}^{2}\end{array}$ & LF nu & $\begin{array}{c}\mathrm{HF}(0.15-0.4 \mathrm{~Hz}) \\
\mathrm{ms}^{2}\end{array}$ & HF nu & $\mathrm{LF} / \mathrm{HF}$ \\
\hline $\begin{array}{c}95 \\
(11)\end{array}$ & $\begin{array}{c}36 \\
(18)\end{array}$ & $\begin{array}{c}1457 \\
(1946)\end{array}$ & $\begin{array}{c}605 \\
(835)\end{array}$ & $\begin{array}{c}75.0 \\
(15.2)\end{array}$ & $\begin{array}{c}242 \\
(522)\end{array}$ & $\begin{array}{c}18.7 \\
(10.4)\end{array}$ & $\begin{array}{c}5.86 \\
(4.64)\end{array}$ \\
\hline
\end{tabular}

beats per minute, and the mean heart rate after exercise was 102 beats per minute.

When pre-exercise was compared to post exercise, a significantly higher heart rate was recorded, with a significantly lower SD RR, as reported in Table 1. In the frequency domain, total power, and both low and high frequency components were significantly reduced following exercise. However, when frequency data were normalised (to take into account the reduction in spectrum total power), an increased low frequency component, and a decreased high frequency component was recorded following exercise. A significant increase in the low-to-high frequency ratio (LF/HF) was also recorded following exercise (see Table 1).
The differences in both low and high frequency components of HRV recorded post exercise were plotted against the difference in heart rate recorded following exercise, as shown in figure2. Linear regression was used to quantify the association between exercise-induced changes in HRV frequency components and the tachycardia recorded following exercise. Weak associations were recorded for both low and high frequency components $\left(R^{2}\right.$ values of 0.0864 and 0.073 , respectively), as shown in Fig. 2.

\section{Discussion}

In this study, the autonomic control of the postexercise tachycardia has been investigated in trained

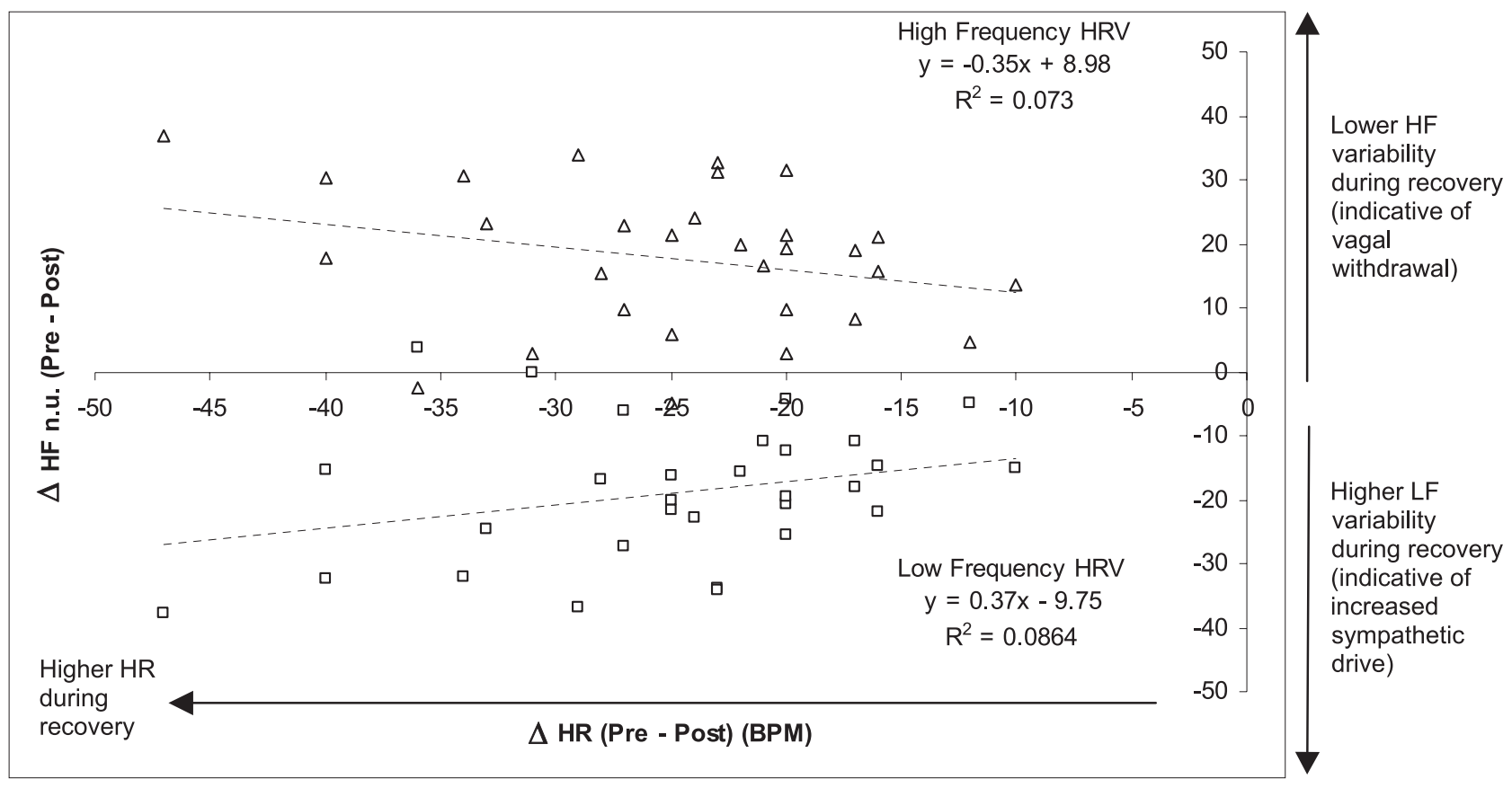

Fig. 2. Relation between the difference in heart rate before (Pre-) and after (Post) exercise and the difference in both high (HF) and low (LF) frequency components of heart rate variability before (Pre-) and after (Post) exercise. 
juveniles by the use of the non-invasive HRV technique. Heart rate variability is a common tool used in the study of Human autonomic control, and exercise physiologists have used HRV to examine the autonomic contribution to the recovery of resting heart rates following exercise. The differences in HRV before and after exercise are well documented for trained and untrained adults of both genders, across a broad age range. However, the current study uniquely applies these methods to a population of highly trained juvenile cyclists - a population in which these data are currently unavailable. Therefore, this study is unique in that it reports, in trained juveniles, an increase in the normalized low frequency components, and a decrease in the normalized high frequency components of HRV following a single bout of maximal exercise, and significantly reduced variability in the time domain (SD RR). Using linear regression, this study uniquely reports a very weak association between the decrease in cardiac vagal control (high frequency component) and the increase in heart rate during recovery. This study also uniquely reports a very weak association between the increase in the low frequency component of HRV and the increase in heart rate during recovery.

The decrease in time domain variability is characteristic of an elevated, but stable mean heart rate. This is consistent with both a decreased HF component and an increased LF component of HRV. Collectively, this supports the notion that the sympathetic branch of the autonomic nervous system is dominant during the early stages of recovery from exercise. The magnitude of HF reduction, when expressed as a proportion of total power, is indicative of vagal withdrawal, thus it was reasonable to hypothesize an association between the magnitude of vagal withdrawal and the increase in mean heart rate. In the current study, this was investigated using regression, where the difference in HF variability between pre- and post exercise was plotted as a function of the difference in heart rate between pre- and post exercise (see figure 2). However, there was only a weak association between these variables, suggesting the increased mean heart rate during the early stages of recovery was independent of the magnitude of vagal withdrawal.

Although the origins of low frequency heart rate variability are diverse [14], a proportion of the increased LF component measured post exercise in the current study is indicative of an elevated sympathetic drive. Again, it was reasonable to hypothesize that an association between the increased LF component post exercise would be associated with an elevated heart rate, but the association between $\Delta \mathrm{LF}$ and $\Delta \mathrm{HR}$ was weak (see figure 2). The increased LF component reported in the current study is not a consistent finding of similar studies, where increases [10, 20 -22] or no changes $[9,12,15]$ have been previously reported.
An elevated LF component may indicate an increased sympathetic outflow to the heart such that an increased cardiac sympathetic drive may be required in the postexercise recovery period in order to compensate for a re-distributed blood volume. A prolonged elevation in the perfusion of skeletal muscle during recovery may require an increased cardiac output in order to maintain blood pressure homeostasis, and this is probably brought about by increases in both heart rate and ventricular ejection fraction. However, the origin of the LF component may reside in the baroreceptor reflex $[19,23]$ and thus increases in the post-exercise period may reflect an exercise-induced change in baroreceptor sensitivity $[7,24]$. No data are currently available for exercise-induced changes in baroreceptor sensitivity in trained juveniles, therefore the origins and mechanism responsible for the elevated low frequency component recorded in the current study, requires further investigation.

There is a possibility that the regression of a frequency domain measure with a time domain measure contains some calculation artefact. However, there is no guarantee of a reciprocal change in $\mathrm{R}-\mathrm{R}$ interval and the frequency components of HRV given that the frequency domain components of HRV provide a measure of the strength of oscillation, irrespective of whether the heart rate is high or low. For example, high frequency variability may be abolished with administration of atropine while mean heart rate remains unchanged [25]. Equally, high frequency variability may increase with a respiratory acidosis with either minimal or no change in R-R interval $[26,27]$.

The HRV high frequency range used in the current study $(0.15-0.4 \mathrm{~Hz})$ is consistent with guidelines for the analysis of HRV. This range includes normal resting respiratory frequencies, including those measured in the current study (Pre: $0.187 \mathrm{~Hz}$, Post: $0.238 \mathrm{~Hz}$ ). However, there is spontaneous variation in both respiratory depth and rate at rest, and this may influence the magnitude of respiratory sinus arrhythmia (RSA) and the frequency distribution of R-R intervals. Imposing a fixed frequency breathing rate during HRV analysis may affect both RSA and respiratory variables (e.g. $\dot{\mathrm{V}} \mathrm{O}_{2}$ and $\dot{\mathrm{V}} \mathrm{CO}_{2}$ ) and it is known that a respiratory acidosis has significant effects on the frequency components of HRV [26]. It has been suggested that higher heart rates during recovery allow the clustering of heart beats during inhalation [13]. This potentially leads to an increase in the efficiency of ventilation by closer matching of pulmonary perfusion to alveolar ventilation, and reducing intrapulmonary shunt during exhalation. Clustering of heart beats during inhalation has been demonstrated following exercise [13] although this observation was independent of any change in HRV, and therefore, potentially independent of autonomic control. The increase in heart rate during 
inhalation post exercise may indicate a cardiac reflex independent of the vagus nerve (therefore independent of high frequency variability measures), whereby an inhalation-induced increase in venous return may initiate more rapid SA node depolarisation [28]. A dissociation of high frequency HRV and respiratory sinus arrhythmia has been reported during exposure to normoxic hypercapnia [26] and during a head-up tilt procedure [29]. However, in the present study, a reduced high frequency component of HRV following exercise is inconsistent with inhalation-induced cardio-acceleration, and we were unable to detect any clustering of heart beats synchronous to the ventilatory cycle. Therefore it remains to be determined if clustering of heart beats is occurring, when a combination of elevated heart rate and reduced high frequency variability following exercise occurs in trained juveniles.

We have chosen not to include a 'control' group in the current study, maintaining a focus on the differences between measures of HRV before and after exercise in a group of highly trained juvenile cyclists. We accept that there are valid comparisons to be made between two potential control groups: firstly, age and gender matched untrained juveniles; secondly, gender matched trained adults. Data are readily available for the latter [9-13], however, there remains a paucity of data on untrained juveniles. The maximal exercise testing protocol used in the current study is not suitable for untrained children, and we were limited by both safety and ethical considerations concerning the maximal testing of juveniles who are not regularly active. Therefore, a limitation of the data presented in the current study is that a clear comparison with a control group of age and gender matched, but untrained juveniles, cannot be made.

In summary, this study examined the autonomic control of an elevated heart rate following exercise, in a group of elite juvenile cyclists. The directional changes in frequency components of the HRV power spectrum were consistent with both a reduced vagal control and an increased sympathetic drive. However, we were unable to show clear association between these changes in frequency components of HRV and the post exercise elevation in heart rate.

\section{References}

1. Akselrod S, Gordon D, Madwed J, et al. Power spectrum analysis of heart rate fluctuations: a quantitative probe of beat - to - beat cardiovascular control. Science 1981; 213: 220-2.

2. Eckberg D, Sleight $\mathrm{P}$ (Eds) Human baroreflexes in health and disease. Oxford, UK: Clarendon Press, 1992.

3. Pardo Y, Merz CN, Paul-Labrador M, et al. Heart rate variability reproducibility and stability using commercially available equipment in coronary artery disease with daily life myocardial ischemia. Am J Cardiol 1996; 78: 866-70.

4. Huikuri H, Makikallio T, Airaksinen J, et al. Measurement of heart rate variability: A clinical tool or a research toy? J Am Coll Cardiol 1999; 34: 1878-83.
5. Aubert A, Seps B, Beckers F. Heart rate variability in athletes. Sports Med 2003; 33: 889-919.

6. Javorka M, Zila I, Balharek T, Javorka K. Heart rate recovery after exercise: relations to heart rate variability and complexity. Braz J Med Biol Res 2002; 35: 991-1000.

7. Raczak G, Pinna G, LaRovere M, et al. (2005) Cardiovagal response to acute mild exercise in young healthy subjects. Circulation J 2005; 69: 976-80.

8. Takahashi T, Okada A, Saitoh T, et al. Difference in human cardiovascular response between upright and supine recovery from upright cycle exercise. Eur J Appl Physiol 2000; 81: 233-9.

9. James D, Barnes A, Lopes P, Wood D. Heart rate variability: Response following a single bout of interval training. Int $J$ Sports Med 2002; 23: 247-51.

10. Parekh A, Lee C. Heart rate variability after isocaloric exercise bouts of different intensities. Med Sci Sports Exerc 2005; 37: 599-605.

11. Yamamoto K, Miyachi M, Saitoh T, et al. Effects of endurance training on resting and post-exercise cardiac autonomic control. Med Sci Sports Exerc 2001; 33: 1496-502.

12. Brown S, Brown, J. Resting and post-exercise cardiac autonomic control in trained Masters Athletes. J Physiol Sci 2007; 57: 23-9.

13. Brown S. Dissociation of respiratory sinus arrhythmia and high frequency heart rate variability following exercise. Med Sport 2010; 14: 43-9.

14. Stahle A, Nordlander R, Bergfeldt L. Aerobic group training improves exercise capacity and heart rate variability in elderly patients with a recent coronary event. Eur Heart J 1999; 20: 1638-46.

15. Perini R, Fisher N, Veicsteinas A, Pendergast D. Aerobic training and cardiovascular responses at rest and during exercise in older men and women. Med Sci Sports Exerc 2002; 34: 700-8.

16. Uusitalo A, Laitinen T, Vaisanen SB, et al. Effects of endurance training on heart rate and blood pressure variability. Clin Physiol Funct Imag 2002; 22: 173-9.

17. Uusitalo A, Laitinen T, Vaisanen SB, et al. Physical training and heart rate and blood pressure variability: a 5-yr randomized trial. Am J Physiol - Heart Circulatory Physiol 2004; 286: H1821-26.

18. Levy W, Cerqueira M, Harp G, et al. Effect of endurance exercise training on heart rate variability at rest in healthy young and older men. Am J Cardiol 1998; 82: 1236-41.

19. Malpas S. Neural influences on cardiovascular variability: possibilities and pitfalls. Am J Physiol - Heart Circulatory Physiol 2002; 282: H6-20.

20. Brown S, Ryan H, Brown J. Non-invasive measures of cardiac autonomic control following high intensity exercise in endurance trained older male cyclists. NZ J Sports Med 2007; 35: 28-34.

21. Goulopoulou S, Heffernan K, Fernhall B, et al. Heart Rate Variability during Recovery from a Wingate Test in Adolescent Males. Med Sci Sports Exerc 2006; 38: 875-81.

22. Hautala A, Tulppo MP, Makikallio TH, et al. Changes in cardiac autonomic regulation after prolonged maximal exercise. Clin Physiol, 2001; 21: 238-45.

23. Boutcher S, Stein P. Association between heart rate variability and training response in sedentary middle-aged men. Eur $J$ Appl Physiol 1995; 70: 75-80.

24. Somers VK, Conway J, Johnston J, Sleight P. Effects of endurance training on baroreflex sensitivity and blood pressure in borderline hypertension. Lancet 1991; 337: 1363-8.

25. Ito S, Sasano H, Sasano N, et al. Vagal nerve activity contributes to improve the efficiency of pulmonary gas exchange in hypoxic humans. Exp Physiol 2006; 91: 935-41.

26. Brown S, Mundel T, Brown J. Cardiac vagal control and respiratory sinus arrhythmia during hypercapnia in humans. $J$ Physiol Sci 2007; 57: 337-42.

27. Tzeng YC, Larsen PD, Galletly DC. Effects of hypercapnia and hypoxemia on respiratory sinus arrhythmia in conscious humans during spontaneous respiration. Am J Physiol - Heart Circulatory Physiol 2007; 292: H2397-407. 
28. Blain G, Meste O, Bermon S. Influences of breathing patterns on respiratory sinus arrhythmia in humans during exercise. Am J Physiol - Heart Circulatory Physiol 2005; 288: H887-95.

29. Brown S, Bryant M, Mündel T., Stannard S.R. Human ventilatory efficiency and respiratory sinus arrhythmia during head-up tilt. J Physiol Pharmacol 2009; 59: 771-80.

Received: May 18, 2011

Accepted: July 20, 2011

Published: July 28, 2011

Address for correspondence:

S. J. Brown

Private bag 102-904

Institute of Food, Nutrition and Human Health,

Massey University,

Albany.

Auckland, NZ.

Telephone: 6494140800 ext 41101

Fax: 6494439640

Email: s.j.brown@massey.ac.nz

Aaron Raman: aaronraman89@gmail.com Zach Schlader: z.j.schlader@massey.ac.nz

Stephen Stannard: s.stannard@massey.ac.nz 